\title{
Parton Showers beyond Leading Logarithmic Accuracy
}

\author{
Mrinal Dasgupta, ${ }^{1}$ Frédéric A. Dreyer, ${ }^{2}$ Keith Hamilton, ${ }^{3}$ Pier Francesco Monni®${ }^{4}$ \\ Gavin P. Salam $\odot,{ }^{2, *}$ and Grégory Soyez ${ }^{5}$ \\ ${ }^{1}$ Consortium for Fundamental Physics, School of Physics and Astronomy, \\ University of Manchester, Manchester M13 9PL, United Kingdom \\ ${ }^{2}$ Rudolf Peierls Centre for Theoretical Physics, Parks Road, Oxford OX1 3PU, United Kingdom \\ ${ }^{3}$ Department of Physics and Astronomy, University College London, London WCIE 6BT, United Kingdom \\ ${ }^{4}$ CERN, Theoretical Physics Department, CH-1211 Geneva 23, Switzerland \\ ${ }^{5}$ Institut de Physique Théorique, Université Paris-Saclay, CNRS, CEA, F-91191 Gif-sur-Yvette, France
}

(Received 3 March 2020; revised 15 May 2020; accepted 26 June 2020; published 28 July 2020)

\begin{abstract}
Parton showers are among the most widely used tools in collider physics. Despite their key importance, none so far have been able to demonstrate accuracy beyond a basic level known as leading logarithmic order, with ensuing limitations across a broad spectrum of physics applications. In this Letter, we propose criteria for showers to be considered next-to-leading logarithmic accurate. We then introduce new classes of shower, for final-state radiation, that satisfy the main elements of these criteria in the widely used large- $N_{C}$ limit. As a proof of concept, we demonstrate these showers' agreement with all-order analytical next-to-leading logarithmic calculations for a range of observables, something never so far achieved for any parton shower.
\end{abstract}

DOI: 10.1103/PhysRevLett.125.052002

High-energy particle collisions produce complex hadronic final states. Understanding these final states is of crucial importance in order to extract maximal information about the underlying energetic scattering processes and the fundamental Lagrangian of particle physics. To do so, there is ubiquitous reliance on general purpose Monte Carlo event generators [1], which provide realistic simulations of full events. A core component of general purpose Monte Carlo event generators is the parton shower, a subject of much recent research [2-28]. Partons refer to quarks and gluons, and a shower aims to encode the dynamics of parton production between the high-energy scattering (e.g., production of electroweak or new-physics states) and the low scale of hadronic quantum chromodynamics (QCD), at which experimental observations are made.

Typically parton showers are built using a simple Markovian algorithm that takes an $n$-parton state and stochastically maps it to an $(n+1)$-parton state. The iteration of this procedure, e.g., starting from a two-parton state, builds up events with numerous partons. A further step, hadronization, then maps the partons onto a set of hadrons. Even though this last step involves modeling

Published by the American Physical Society under the terms of the Creative Commons Attribution 4.0 International license. Further distribution of this work must maintain attribution to the author(s) and the published article's title, journal citation, and DOI. Funded by SCOAP ${ }^{3}$.
$[29,30]$, many of the features of the resulting events are driven by the parton shower component which is, in principle, within the realm of calculations in perturbative QCD. This is because the showering occurs at momentum scales where the strong coupling, $\alpha_{s}$, is small.

Much of collider physics, experimental and theoretical [31-34], is moving towards high precision, especially in view of large volumes of data collected so far at CERN's Large Hadron Collider. On the theoretical front many of the advances either involve approximations with a small number of partons, or else are specific to individual observables. Parton showers, in contrast, use a single algorithm to describe arbitrary observables of any complexity. This versatility comes at a cost: lesser accuracy for any specific observable and, quite generally, at best only limited knowledge [35-38] of what the accuracy even is for a given observable. In fact there is currently no readily accepted criterion for categorizing the accuracy of parton showers. One novel element that we introduce in this Letter is therefore a set of criteria for doing so.

The role of showers is to reproduce emissions across disparate scales. Our first criterion for accuracy starts by structuring this phase space: there are three phase space variables per emission, and two of them (e.g., energy and angle) are associated with logarithmic divergences in the product of squared matrix element and phase space. We define leading logarithmic (LL) accuracy to include a condition that the shower should generate the correct effective squared tree-level matrix element in a limit where every pair of emissions has distinctly different values for 
both logarithmic variables. At next-to-leading logarithmic (NLL) accuracy, we further require that the shower generate the correct squared tree-level matrix element in a limit where every pair of emissions has distinctly different values for at least one of the logarithmic variables (or some linear combination of their logarithms). Beyond NLL accuracy we would consider configurations with a pair of emissions (or multiple pairs) both of whose logarithmic variables are similar.

To help make this discussion concrete, let us consider showers that are not NLL accurate according to this criterion: angular ordered showers [39-41] do not reproduce the matrix element for configurations ordered in energy, but with commensurate angles, and this is associated with their inability to correctly predict $\alpha_{s}^{n} L^{n}$ (NLL) effects for nonglobal observables [36]. Transverse-momentum $\left(k_{t}\right)$ ordered showers with dipole-local recoil $[2,3,5,11,42,43]$ do not reproduce matrix elements for configurations ordered in angle but with commensurate transverse momenta, because of the way they assign transverse recoil [37]. As a result they fail to reproduce NLL effects in global observables such as jet broadenings. Showers that omit spin correlations fail to reproduce (the azimuthal structure of) matrix elements for configurations ordered in angle but with commensurate energies [44-46], and associated NLL terms.

Our second criterion for logarithmic accuracy tests, among other things, the overall correctness of virtual corrections. For showers that intertwine real and virtual corrections directly through unitarity, once the generation of tree-level matrix elements is set, there is only one (single-emission) degree of freedom that remains, namely the choice of scale and scheme for the strong coupling for each emission, as a function of its kinematics. To claim NLL accuracy, we will require the resulting shower to reproduce known analytical NLL resummations across recursively infrared and collinear safe (rIRC) [47] global and nonglobal two-scale observables as well as (subjet) multiplicities.

The challenge that we concentrate on here is to formulate showers that can handle each of two regions correctly: the energy-ordered, commensurate-angle region; and the angular-ordered, commensurate $k_{t}$ region. Recall that existing $k_{t}$ and angular-ordered showers can each handle one of these limits, but not both. Strictly, full NLL accuracy also requires attention to the angular-ordered, commensurate energy region. However, given that general solutions for the required spin correlations are known to exist [45,48-50], and that they affect only a small subset of observables, we postpone their study to future work. For now, we also restrict our attention to final-state showers (i.e., leptonlepton collisions), massless quarks and the large- $N_{C}$ limit. Our guiding principle will be that soft emissions should not affect, or be affected by, subsequent emissions at disparate rapidities.
The two classes of shower that we develop both consider emissions from colour dipoles. We consider a continuous family of shower evolution variables $v$, parameterised by a quantity $\beta$ in the range $0 \leq \beta<1$, where $\beta=0$ corresponds to transverse-momentum ordering. The phase space involves two further variables besides $v$ : a pseudorapiditylike variable within the dipole, $\bar{\eta}$, and an azimuthal angle $\phi$.

We start with a shower with dipole-local recoil (the PanLocal shower). Its mapping for emission of momentum $p_{k}$ from a dipole $\left\{\tilde{p}_{i}, \tilde{p}_{j}\right\}$ is

$$
\begin{gathered}
p_{k}=a_{k} \tilde{p}_{i}+b_{k} \tilde{p}_{j}+k_{\perp}, \\
p_{i}=a_{i} \tilde{p}_{i}+b_{i} \tilde{p}_{j}-f k_{\perp}, \\
p_{j}=a_{j} \tilde{p}_{i}+b_{j} \tilde{p}_{j}-(1-f) k_{\perp},
\end{gathered}
$$

where $k_{\perp}=k_{t}\left[n_{\perp, 1} \cos \phi+n_{\perp, 2} \sin \phi\right]$, with $n_{\perp, m}^{2}=-1$, $n_{\perp, m} \cdot \tilde{p}_{i / j}=0(m=1,2), n_{\perp, 1} \cdot n_{\perp, 2}=0$, and

$$
k_{t}=\rho v e^{\beta|\bar{\eta}|}, \quad \rho=\left(\frac{s_{\tilde{l}} S_{\tilde{\jmath}}}{Q^{2} s_{\tilde{\imath} \tilde{\jmath}}}\right)^{\frac{\beta}{2}} .
$$

Here $s_{\tilde{\imath} \tilde{j}}=2 \tilde{p}_{i} \cdot \tilde{p}_{j}, s_{\tilde{\imath}}=2 \tilde{p}_{i} \cdot Q$, and $Q$ is the total event momentum. The light-cone components of $p_{k}$ are given by

$$
a_{k} \equiv \sqrt{\frac{S_{\tilde{\jmath}}}{s_{\tilde{\imath} \tilde{\jmath}} S_{\tilde{l}}}} k_{t} e^{+\bar{\eta}}, \quad b_{k} \equiv \sqrt{\frac{S_{\tilde{l}}}{s_{\tilde{\imath} \tilde{\jmath}} S_{\tilde{\jmath}}}} k_{t} e^{-\bar{\eta}},
$$

The quantity $f$ in Eq. (1) determines how transverse recoil is shared between $p_{i}$ and $p_{j}$, cf. below. The $a_{i}, b_{i}, a_{j}, b_{j}$ are fully specified by the requirements $p_{i / j}^{2}=0,\left(p_{i}+p_{j}+\right.$ $\left.p_{k}\right)=\left(\tilde{p}_{i}+\tilde{p}_{j}\right)$ and $p_{i}=\tilde{p}_{i}$ for $k_{t} \rightarrow 0$ and are given explicitly in Sec. 1 of the Supplemental Material [51].

In the event center-of-mass frame, $\bar{\eta}=0$ corresponds to a direction equidistant in angle from $\tilde{p}_{i}$ and $\tilde{p}_{j}$. For soft-collinear emissions, the physical pseudorapidity, $\eta=-\ln \tan (\theta / 2)$, with respect to the emitter is $\eta=|\bar{\eta}|+(1 / \beta) \ln \rho$. Soft-collinear emissions from distinct dipoles but with the same $\ln v$ fall onto common contours in the Lund plane [52], $k_{t}=v e^{\beta|\eta|}$.

For $e^{+} e^{-} \rightarrow$ hadrons, the shower starts from a twoparton $q \bar{q}$ state, $\mathcal{S}_{2}$. The probability of evolving from $\mathcal{S}_{n} \rightarrow \mathcal{S}_{n+1}$ in a given slice $d \ln v$ of evolution variable is

$$
\begin{aligned}
\frac{d \mathcal{P}_{n \rightarrow n+1}}{d \ln v}= & \sum_{\operatorname{dipoles}\{\tilde{\imath} \tilde{\jmath}\}} \int d \bar{\eta} \frac{d \phi}{2 \pi} \frac{\alpha_{s}\left(k_{t}\right)+K \alpha_{s}^{2}\left(k_{t}\right)}{\pi} \\
& \times\left[g(\bar{\eta}) a_{k} P_{\tilde{\imath} \rightarrow i k}\left(a_{k}\right)+g(-\bar{\eta}) b_{k} P_{\tilde{\jmath} \rightarrow j k}\left(b_{k}\right)\right],
\end{aligned}
$$

with a function $g(\bar{\eta})$ that satisfies $g(\bar{\eta})+g(-\bar{\eta})=1$, has $g(\bar{\eta})=0$ (1) for sufficiently negative (positive) $\bar{\eta}$, and smoothly transitions around $\bar{\eta}=0$. The $P_{\tilde{\imath} \rightarrow i k}(z)$ are first-order splitting functions [53-55], normalized so that 

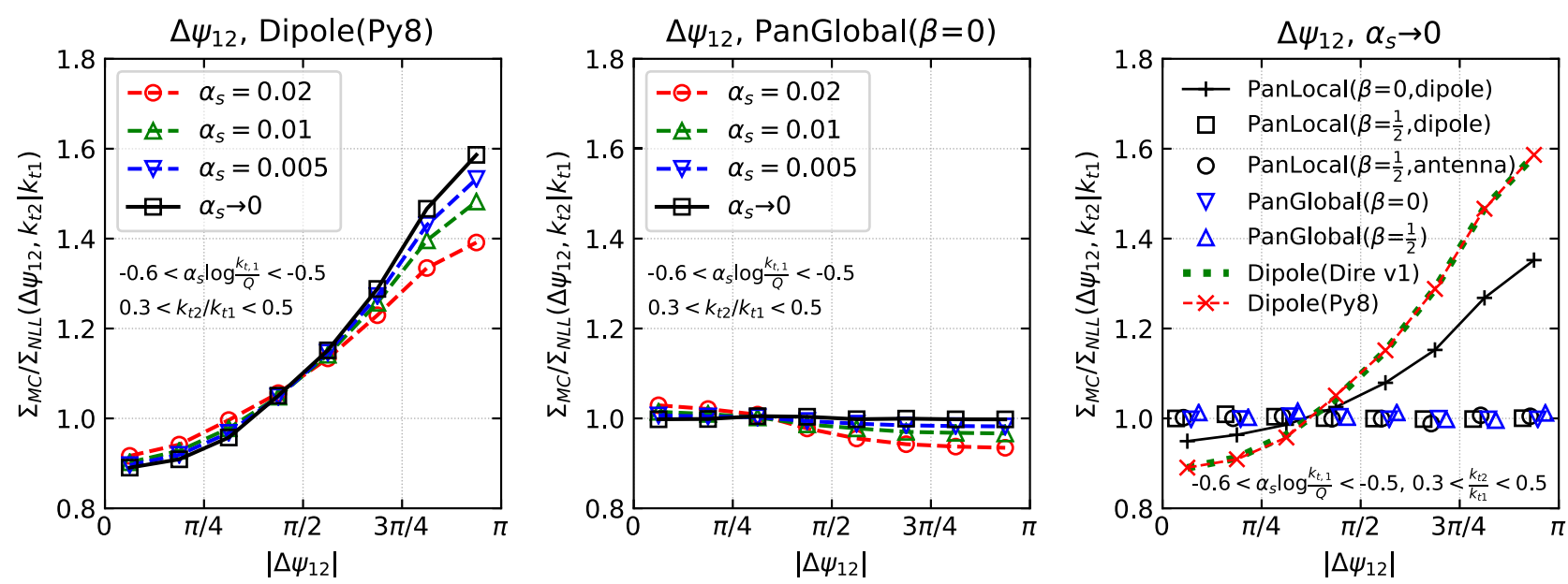

FIG. 1. Left: distribution for the difference in azimuthal angle between the two highest- $k_{t}$ primary Lund declusterings in the PYTHIA8 dipole shower algorithm, normalized to the NLL result [51,66], Sec. 4 of the Supplemental Material; successively smaller $\alpha_{s}$ values keep fixed $\alpha_{s} \ln k_{t 1}$. Middle: the same for the $\operatorname{PanGlobal}(\beta=0)$ shower. Right: the $\alpha_{s} \rightarrow 0$ limit of the ratio for multiple showers. This observable directly tests part of our NLL (squared) matrix-element correctness condition. A unit value for the ratio signals success.

$\lim _{z \rightarrow 0} z P_{\tilde{\imath} \rightarrow i k}(z)=2 C \quad$ with $\quad C=C_{F}=C_{A} / 2=(4 / 3)$ (our large- $N_{C}$ approximation, augmented [56] with $\left.n_{f}=5\right)$. The specific choice of $g(\bar{\eta})$ is not critical here, while the splitting functions are standard. Both are detailed in Sec. 1 of the Supplemental Material [51]. The $\overline{\mathrm{MS}}$ coupling, $\alpha_{s}\left(k_{t}\right)$, needs at least two-loop running, and $K=$ $(1 / 2 \pi)\left\{\left[(67 / 18)-\left(\pi^{2} / 6\right)\right] C_{A}-(5 / 9) n_{f}\right\}$ [57].

The PanLocal shower comes in two variants. In a dipole variant, inspired by many earlier dipole showers [2,5,11], the $P_{\tilde{\imath} \rightarrow i k}\left(a_{k}\right)\left[P_{\tilde{\jmath} \rightarrow j k}\left(b_{k}\right)\right]$ term of Eq. (4) is associated with the choice $f=1(f=0)$ in Eq. (1). In an antenna variant, inspired by Refs. $[3,43]$, we take a common $f(\bar{\eta})$ for both terms and set $f(\bar{\eta})=g(\bar{\eta})$.

A key difference relative to earlier showers is that our transition in transverse recoil assignment between $i$ and $j$ takes place at $\bar{\eta} \simeq 0$, i.e., equal angles between the $\tilde{p}_{i}$ and $\tilde{p}_{j}$ directions in the event center-of-mass frame (note similarities with DEDUCTOR [9]). This differs from the common choice of a transition in the middle of the dipole center-ofmass frame. Our choice ensures that a given emission will not induce transverse recoil in earlier, lower-rapidity emissions. Additionally, we require $\beta>0$ in the definition of the ordering variable, Eq. (2). This causes emissions at commensurate $k_{t}$ and widely separated in $|\eta|$ to be effectively produced in order of increasing $|\eta|$, so that any significant $k_{t}$ recoil is always taken from the extremities of a (hard) $q g\{\ldots\} g \bar{q}$ dipole chain. Together, these two elements provide a solution to the problem observed in Ref. [37], i.e., that recoil assignment in common dipole showers causes multigluon emission matrix elements to be incorrect in the limit of similar $k_{t} \mathrm{~s}$ and disparate angles, starting from $\alpha_{s}^{2}$, leading to incorrect NLL terms.

Note that with dipole-local recoil, NLL correctness also requires $\beta<1$, because with $\beta \geq 1$ the kinematic constraint associated with fixed dipole mass means that a first emission cuts out regions of phase space for a second emission at similar $\ln v$.

A second class of shower can be constructed with global, i.e., event-wide recoil (the PanGlobal shower). It can be formulated in largely the same terms as the dipole-local recoil shower, but with a two-step recoil procedure. In the first step one sets

$$
\begin{gathered}
\bar{p}_{k}=a_{k} \tilde{p}_{i}+b_{k} \tilde{p}_{j}+k_{\perp}, \\
\bar{p}_{i}=\left(1-a_{k}\right) \tilde{p}_{i}, \\
\bar{p}_{j}=\left(1-b_{k}\right) \tilde{p}_{j} .
\end{gathered}
$$

The second step is to apply a boost and rescaling to the full event (including the $\bar{p}_{i, j, k}$ momenta) so as to obtain final momenta $\{p\}$ whose sum gives $Q$. This approach assigns transverse recoil dominantly to the most energetic particles in the event. Thus emission from a hard $q g\{\ldots\} g \bar{q}$ dipole string transfers its recoil mostly to the hard $q$ and $\bar{q}$ ends. This ensures that one reproduces a pattern of independent emission for commensurate- $k_{t}$ and angular-ordered gluons, while also retaining the correct (dipole) pattern for energy-ordered, commensurate angles. This holds even for $\beta=0$, i.e., with $k_{t}$ ordering. Values of $\beta \geq 1$ remain problematic, however. Note that the PanGlobal shower has power-suppressed routes to highly collimated events. These compete with normal Sudakov suppression, as observed also for PYTHIA8 [37]. We have verified that such effects are small even at the very edges of future (FCC-hh [58]) phenomenologically accessible regions. Nevertheless, ultimately one may wish to explore alternative global recoil schemes.

The next step is to compare our showers to NLL observables. Relative to earlier attempts at such comparisons 

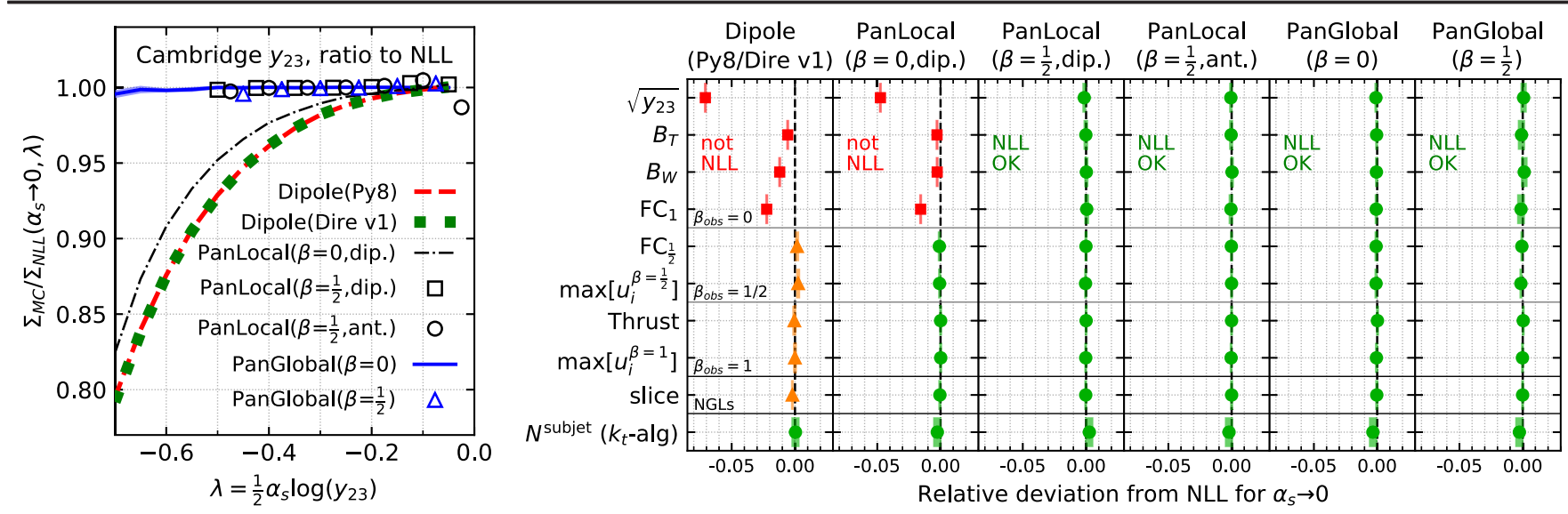

FIG. 2. Left: ratio of the cumulative $y_{23}$ distribution from several showers divided by the NLL answer, as a function of $\alpha_{s} \ln y_{23} / 2$, for $\alpha_{s} \rightarrow 0$. Right: summary of deviations from NLL for many shower and observable combinations [either $\Sigma_{\text {shower }}\left(\alpha_{s} \rightarrow 0, \alpha_{s} L=\right.$ $-0.5) / \Sigma_{\mathrm{NLL}}-1$ or $\left.\left[N_{\text {shower }}^{\text {subjet }}\left(\alpha_{s} \rightarrow 0, \alpha_{s} L^{2}=5\right) / N_{\mathrm{NLL}}^{\text {subjet }}-1\right] / \sqrt{\alpha_{s}}\right]$. Red squares indicate clear NLL failure; amber triangles indicate NLL fixed-order failure that is masked at all orders; green circles indicate that all NLL tests passed.

[59], a critical novel aspect is how we isolate the structure of NLL terms $\alpha_{s}^{n} L^{n}$. For each given observable $v$, with $L=\ln v$, we consider the ratio to the true NLL result in the limit $\alpha_{s} \equiv \alpha_{s}(Q) \rightarrow 0$ with fixed $\alpha_{s} L$. This helps us isolate the NLL terms from yet higher-order contributions, which vanish in that limit. Numerically, a parton shower cannot be run in the $\alpha_{s} \rightarrow 0$ limit for fixed $\alpha_{s} L$. However, with suitable techniques [51] (Sec. 6), [60-62], one can run multiple small values of $\alpha_{s}$ and extrapolate to $\alpha_{s}=0$. We examine not just our showers, but also our implementations of two typical $k_{t}$-ordered shower algorithms with dipolelocal recoil, those of PYTHIA8 [2] and DIRE V1 [11] [with the $\alpha_{s}+K \alpha_{s}^{2}$ choice as in Eq. (4)].

A first test concerns the multiple-emission matrix element. We have constructed our showers specifically so that they reproduce the squared matrix elements in the limits discussed above that are relevant for NLL accuracy. A simple observable for testing this is to consider the two highest- $k_{t}$ Lund-plane primary declusterings $[63,64]$ with transverse momenta $k_{t 1}$ and $k_{t 2}$ (originally defined for hadronic collisions, the $e^{+} e^{-}$analogue is given in Sec. 4 of the Supplemental Material [51] and implemented with FASTJET [65]). The $\alpha_{s} \rightarrow 0$ limit for fixed $\alpha_{s} L$ $\left(L=\ln k_{t 1} / Q\right)$, ensures that the two declusterings are soft and widely separated in Lund-plane pseudorapidity $\eta$ (which spans $|\eta| \lesssim|L| \sim 1 / \alpha_{s}$ ). In this limit the full matrix element reduces to independent emission and so the difference of azimuthal angles between the two emissions, $\Delta \psi_{12}$, should be uniformly distributed, for any ratio $k_{t 2} / k_{t 1}$ (recall that strongly angular-ordered soft emission is not affected by spin correlations). We consider the $\Delta \psi_{12}$ distribution in Fig. 1.

The left-hand plot of Fig. 1 shows the PYTHIA8 dipole algorithm (not designed as NLL accurate), while the middle plot shows our PanGlobal shower with $\beta=0$. The dipole result is clearly not independent of $\Delta \psi_{12}$ for $\alpha_{s} \rightarrow 0$, with over $60 \%$ discrepancies, extending the fixed-order conclusions of Ref. [37]. The discrepancy is only $\simeq 30 \%$ for $g g$ events (not shown in Fig. 1), and the difference would, e.g., skew machine learning [67] for quark versus gluon discrimination. PanGlobal is independent of $\Delta \psi_{12}$. The righthand plot shows the $\alpha_{s} \rightarrow 0$ limit for multiple showers. The overall pattern is as expected: PanLocal works for $\beta=0.5$, but not $\beta=0$, demonstrating that with $k_{t}$ ordering it is not sufficient just to change the dipole partition to get NLL accuracy. PanGlobal works for $\beta=0$ and $\beta=0.5$. (Showers that coincide for $\alpha_{s} \rightarrow 0$, e.g., DIRE V1 and PYTHIA8, typically differ at finite $\alpha_{s}$, reflecting NNLL differences.)

Next, we consider a range of more standard observables at NLL accuracy. They include the Cambridge $\sqrt{y_{23}}$ resolution scale [68], two jet broadenings, $B_{T}$ and $B_{W}$ [69], fractional moments, $\mathrm{FC}_{1-\beta_{\mathrm{obs}}}$, of the energy-energy correlations [47], the thrust [70,71], and the maximum $u_{i}=$ $k_{t i} / Q e^{-\beta_{\text {obs }}\left|\eta_{i}\right|}$ among primary Lund declusterings $i$. Each of these is sensitive to soft-collinear radiation as $k_{t} / Q e^{-\beta_{\mathrm{obs}}|\eta|}$, with the $\beta_{\text {obs }}$ values shown in Fig. 2 (right). Additionally, the scalar sum of the transverse momenta in a rapidity slice [72], of full-width 2, is useful to test nonglobal logarithms (NGLs). These observables all have the property that their distribution at NLL can be written as $[47,66,72-74]$

$\Sigma\left(\alpha_{s}, \alpha_{s} L\right)=\exp \left[\alpha_{s}^{-1} g_{1}\left(\alpha_{s} L\right)+g_{2}\left(\alpha_{s} L\right)+\mathcal{O}\left(\alpha_{s}^{n} L^{n-1}\right)\right]$,

where $\Sigma$ is the fraction of events where the observable is smaller than $e^{L}\left(g_{1}=0\right.$ for the rapidity slice $\left.k_{t}\right)$. We also consider the $k_{t}$-algorithm [75] subjet multiplicity [76], Sec. 5 of the Supplemental Material [51].

Figure 2 (left) illustrates our all-order tests of the shower for one observable, $\sqrt{y_{23}}$. It shows the ratio of the $\Sigma$ as 
calculated with the shower to the NLL result, as a function of $\alpha_{s} \ln \sqrt{y_{23}}$ in the limit of $\alpha_{s} \rightarrow 0$. The standard dipole algorithms disagree with the NLL result, by up to $20 \%$. This is non-negligible, though smaller than the disagreement in Fig. 1, because of the azimuthally averaged nature of the $\sqrt{y_{23}}$ observable. In contrast the PanGlobal and PanLocal $(\beta=0.5)$ showers agree with the NLL result to within statistical uncertainties.

Figure 2(right) shows an overall summary of our tests. The position of each point shows the result of $\Sigma_{\text {shower }}\left(\alpha_{s} \rightarrow\right.$ $\left.0, \alpha_{s} L=-0.5\right) / \Sigma_{\mathrm{NLL}}-1$ or $\left[N_{\text {shower }}^{\text {subjet }}\left(\alpha_{s} \rightarrow 0, \alpha_{s} L^{2}=5\right) /\right.$ $\left.N_{\mathrm{NLL}}^{\text {subjet }}-1\right] / \sqrt{\alpha_{s}}$. If it differs from 0 , the point is shown as a red square. In some cases (amber triangles) it agrees with 0 , though an additional fixed-order analysis in a fixedcoupling toy shower [37,51] (Sec. 2) reveals issues affecting NLL accuracy, all involving hitherto undiscovered spurious super-leading logarithmic terms [77].

Green circles in Fig. 2 (right) indicate that the shower and observable combination passes all of our NLL tests, both at all orders and in fixed-order expansions. The four shower algorithms designed to be NLL accurate pass all the tests. These are the PanLocal shower (dipole and antenna variants) with $\beta=\frac{1}{2}$ and the PanGlobal shower with $\beta=0$ and $\beta=\frac{1}{2}$.

To conclude, we have identified two routes towards NLL parton shower accuracy. One involves a modification of the evolution variable and dipole partition, while maintaining dipole-local recoil; the other replaces dipole-local recoil with event-wide recoil. While further work is needed towards phenomenology, the results shown here represent the first time that individual parton showers are demonstrated to be able to reproduce NLL accuracy simultaneously for both nonglobal and a wide set of global observables. It is our hope that these results, together with our NLL criteria and validation framework, can provide the solid foundations needed for future development of logarithmically accurate showers.

We are grateful to Fabrizio Caola, Silvia Ferrario Ravasio, Basem El-Menoufi, Alexander Karlberg, Paolo Nason, Ludovic Scyboz, Rob Verheyen, Bryan Webber, and Giulia Zanderighi for helpful discussions and comments on the manuscript. We thank each other's institutions for hospitality while this work was being carried out. This work has been funded by a Marie Skłodowska Curie Individual Fellowship Contract No. 702610 Resummation4PS (P. M.), by a Royal Society Research Professorship (Grant No. RP\R1\180112) (G. P. S.), by the European Research Council (ERC) under the European Unions Horizon 2020 research and innovation programme (Grant Agreement No. 788223, PanScales) (M. D., K. H., G.P.S., G.S.) and by the Science and Technology Facilities Council (STFC) under Grants No. ST/ P000770/1 (F. D.), No. ST/P000274/1 (K. H.), and No. ST/P000800/1 (M. D.).
"On leave from CNRS, UMR 7589, LPTHE, F-75005 Paris, France and CERN, Theoretical Physics Department, CH1211 Geneva 23, Switzerland.

[1] A. Buckley et al., Phys. Rep. 504, 145 (2011).

[2] T. Sjostrand and P.Z. Skands, Eur. Phys. J. C 39, 129 (2005).

[3] W. T. Giele, D. A. Kosower, and P. Z. Skands, Phys. Rev. D 78, 014026 (2008).

[4] Z. Nagy and D. E. Soper, J. High Energy Phys. 09 (2007) 114.

[5] S. Schumann and F. Krauss, J. High Energy Phys. 03 (2008) 038.

[6] S. Platzer and S. Gieseke, J. High Energy Phys. 01 (2011) 024

[7] S. Jadach, A. Kusina, M. Skrzypek, and M. Slawinska, Nucl. Phys. B, Proc. Suppl. 205-206, 295 (2010).

[8] Z. Nagy and D. E. Soper, J. High Energy Phys. 06 (2012) 044 .

[9] Z. Nagy and D. E. Soper, J. High Energy Phys. 06 (2014) 097.

[10] Z. Nagy and D. E. Soper, J. High Energy Phys. 07 (2015) 119.

[11] S. Hoeche and S. Prestel, Eur. Phys. J. C 75, 461 (2015).

[12] H. T. Li and P. Skands, Phys. Lett. B 771, 59 (2017).

[13] S. Jadach, A. Kusina, W. Placzek, and M. Skrzypek, J. High Energy Phys. 08 (2016) 092.

[14] N. Fischer, S. Prestel, M. Ritzmann, and P. Skands, Eur. Phys. J. C 76, 589 (2016).

[15] Z. Nagy and D. E. Soper, J. High Energy Phys. 10 (2016) 019 .

[16] N. Fischer, A. Lifson, and P. Skands, Eur. Phys. J. C 77, 719 (2017).

[17] S. Hoeche, F. Krauss, and S. Prestel, J. High Energy Phys. 10 (2017) 093.

[18] S. Hoche and S. Prestel, Phys. Rev. D 96, 074017 (2017).

[19] Z. Nagy and D. E. Soper, Phys. Rev. D 98, 014034 (2018).

[20] B. Cabouat and T. Sjstrand, Eur. Phys. J. C 78, 226 (2018).

[21] F. Dulat, S. Hoche, and S. Prestel, Phys. Rev. D 98, 074013 (2018).

[22] S. Plaetzer, M. Sjodahl, and J. Thorn, J. High Energy Phys. 11 (2018) 009.

[23] R. Ángeles Martínez, M. De Angelis, J. R. Forshaw, S. Plaetzer, and M. H. Seymour, J. High Energy Phys. 05 (2018) 044.

[24] J. Isaacson and S. Prestel, Phys. Rev. D 99, 014021 (2019).

[25] H. Brooks and P. Skands, Phys. Rev. D 100, 076006 (2019).

[26] J. R. Forshaw, J. Holguin, and S. Plaetzer, J. High Energy Phys. 08 (2019) 145.

[27] Z. Nagy and D. E. Soper, Phys. Rev. D 100, 074005 (2019).

[28] S. Hoeche and D. Reichelt, arXiv:2001.11492.

[29] A. Buckley, H. Hoeth, H. Lacker, H. Schulz, and J.E. von Seggern, Eur. Phys. J. C 65, 331 (2010).

[30] P. Z. Skands, Phys. Rev. D 82, 074018 (2010).

[31] R. Keith Ellis and G. Zanderighi, in From My Vast Repertoire...: Guido Altarelli's Legacy, edited by A. Levy, S. Forte, and G. Ridolfi (World Scientific, Singapore, 2019), pp. 31-52.

[32] P. Azzi et al., CERN Yellow Reports: Monographs (2019), Vol. 7, pp. 1-220, arXiv:1902.04070. 
[33] M. Cepeda et al., CERN Yellow Reports: Monographs (2019), Vol. 7, pp. 221-584.

[34] X. Cid Vidal et al., CERN Yellow Reports: Monographs (2019), Vol. 7, pp. 585.

[35] G. Marchesini and B. R. Webber, Nucl. Phys. B238, 1 (1984).

[36] A. Banfi, G. Corcella, and M. Dasgupta, J. High Energy Phys. 03 (2007) 050.

[37] M. Dasgupta, F. A. Dreyer, K. Hamilton, P. F. Monni, and G. P. Salam, J. High Energy Phys. 09 (2018) 033.

[38] G. Bewick, S. Ferrario Ravasio, P. Richardson, and M. H. Seymour, J. High Energy Phys. 04 (2020) 019.

[39] G. Marchesini and B. R. Webber, Nucl. Phys. B310, 461 (1988).

[40] G. Corcella, I. G. Knowles, G. Marchesini, S. Moretti, K. Odagiri, P. Richardson, M. H. Seymour, and B. R. Webber, J. High Energy Phys. 01 (2001) 010.

[41] M. Bahr et al., Eur. Phys. J. C 58, 639 (2008).

[42] G. Gustafson and U. Pettersson, Nucl. Phys. B306, 746 (1988).

[43] L. Lonnblad, Comput. Phys. Commun. 71, 15 (1992).

[44] B. R. Webber, Annu. Rev. Nucl. Part. Sci. 36, 253 (1986).

[45] J. C. Collins, Nucl. Phys. B304, 794 (1988).

[46] S. Catani and M. Grazzini, Nucl. Phys. B845, 297 (2011).

[47] A. Banfi, G. P. Salam, and G. Zanderighi, J. High Energy Phys. 03 (2005) 073.

[48] I. G. Knowles, Comput. Phys. Commun. 58, 271 (1990).

[49] Z. Nagy and D. E. Soper, J. High Energy Phys. 07 (2008) 025 .

[50] P. Richardson and S. Webster, Eur. Phys. J. C 80, 83 (2020).

[51] See the Supplemental material at http://link.aps.org/ supplemental/10.1103/PhysRevLett.125.052002 for additional technical details.

[52] B. Andersson, G. Gustafson, L. Lonnblad, and U. Pettersson, Z. Phys. C 43, 625 (1989).

[53] V. N. Gribov and L. N. Lipatov, Yad. Fiz. 15, 781 (1972) [Sov. J. Nucl. Phys. 15, 438 (1972)].

[54] G. Altarelli and G. Parisi, Nucl. Phys. B126, 298 (1977).

[55] Y. L. Dokshitzer, Zh. Eksp. Teor. Fiz. 73, 1216 (1977) [Sov. Phys. JETP 46, 641 (1977)].

[56] A. Behring, K. Melnikov, R. Rietkerk, L. Tancredi, and C. Wever, Phys. Rev. D 100, 114034 (2019).

[57] S. Catani, B. R. Webber, and G. Marchesini, Nucl. Phys. B349, 635 (1991).

[58] A. Abada et al. (FCC Collaboration), Eur. Phys. J. Special Topics 228, 755 (2019).
[59] S. Hoeche, D. Reichelt, and F. Siegert, J. High Energy Phys. 01 (2018) 118.

[60] Y. Hida, X. S. Li, and D. H. Bailey, in 15th IEEE Symposium on Computer Arithmetic (IEEE Computer Society, 2000), pp. 155-162.

[61] A. Lipowski and D. Lipowska, Physica (Amsterdam) 391A, 2193 (2012).

[62] R. Kleiss and R. Verheyen, Eur. Phys. J. C 76, 359 (2016).

[63] F. A. Dreyer, G. P. Salam, and G. Soyez, J. High Energy Phys. 12 (2018) 064.

[64] H. A. Andrews et al., J. Phys. G 47, 065102 (2020).

[65] M. Cacciari, G. P. Salam, and G. Soyez, Eur. Phys. J. C 72, 1896 (2012).

[66] A. Banfi, G. P. Salam, and G. Zanderighi, J. High Energy Phys. 01 (2002) 018.

[67] A. J. Larkoski, I. Moult, and B. Nachman, Phys. Rep. 841, 1 (2020).

[68] Y. L. Dokshitzer, G. D. Leder, S. Moretti, and B. R. Webber, J. High Energy Phys. 08 (1997) 001.

[69] S. Catani, G. Turnock, and B. R. Webber, Phys. Lett. B 295, 269 (1992).

[70] S. Brandt, C. Peyrou, R. Sosnowski, and A. Wroblewski, Phys. Lett. 12, 57 (1964).

[71] E. Farhi, Phys. Rev. Lett. 39, 1587 (1977).

[72] M. Dasgupta and G. P. Salam, J. High Energy Phys. 03 (2002) 017.

[73] Y. L. Dokshitzer, A. Lucenti, G. Marchesini, and G. P. Salam, J. High Energy Phys. 01 (1998) 011.

[74] S. Catani, L. Trentadue, G. Turnock, and B. R. Webber, Nucl. Phys. B407, 3 (1993).

[75] S. Catani, Y. L. Dokshitzer, M. Olsson, G. Turnock, and B. R. Webber, Phys. Lett. B 269, 432 (1991).

[76] S. Catani, Y. L. Dokshitzer, F. Fiorani, and B. R. Webber, Nucl. Phys. B377, 445 (1992).

[77] Such terms, $\left(\alpha_{s} L\right)^{n}\left(\alpha_{s} L^{2}\right)^{p}$ in $\ln \Sigma$, starting typically for $n=3$ (sometimes 2), $p \geq 1$, appear for traditional $k_{t}$ ordered dipole showers for global $\left(\beta_{\text {obs }}>0\right)$ and nonglobal observables [51], Sec. 3. Terms of this kind can generically exist [78-80], but not at leading color or for pure final-state processes with rIRC [47] safe observables. In many cases, the spurious superleading logarithms appear to resum to mask any disagreement with NLL.

[78] N. Brown and W. J. Stirling, Phys. Lett. B 252, 657 (1990).

[79] J. R. Forshaw, A. Kyrieleis, and M. H. Seymour, J. High Energy Phys. 08 (2006) 059.

[80] S. Catani, D. de Florian, and G. Rodrigo, J. High Energy Phys. 07 (2012) 026. 